\title{
Die pseud-athanasianische Disputatio contra Arium. Eine Auseinandersetzung mit »arianischer« Theologie in Dialogform
}

\author{
Annette von Stockhausen
}

\section{Vorbemerkung}

Bereits kurz nach seinem Tod, vielleicht sogar schon in seinen letzten Lebensjahren, ist Athanasius von Alexandrien als »Säule der Orthdoxie«, als das Symbol nizänischer Orthodoxie gerühmt ${ }^{1}$ und als solches im Lauf der Zeit auch vorrangig wahrgenommen worden. Es überrascht daher nicht, daß eine beträchtliche Anzahl an Texten anderer Autoren, von denen zumindest einige im Verdacht standen, häretische Ansichten zu vertreten, im Corpus Athanasianum Zuflucht gefunden haben. Heute, nicht zuletzt dank der Clavis Patrum Graecorum, wissen wir von ungefähr 130 griechisch, lateinisch, koptisch, syrisch, armenisch, georgisch, altkirchenslawisch und arabisch unter dem Namen des Athanasius überlieferten, aber wohl sämtlich nicht von ihm verfaßten Texten, die in ihrer Entstehungszeit von der Lebenszeit des Athanasius bis ins Mittelalter reichen. ${ }^{2}$

Viele der griechischen Texte, die heute für nicht authentisch gehalten werden, wurden bereits von Bernard de Montfaucon am Ende des 17. Jahrhunderts als Pseud-Athanasiana identifiziert. Für den größeren Teil dieser Texte sind die Edition Bernard de Montfaucons ${ }^{3}$ (bzw. ihr 1857 publizierter Nachdruck durch Jean-Paul Migne) und seine die Texte jeweils einleitenden Monita sogar bis

1 Vgl. Greg.Naz., or. 21,26; vgl. auch Cyr., hom.pasch. 8,6 (PG 77,572A = SC 392,100): $\delta$

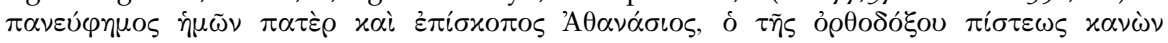
$\dot{\alpha} \iota \dot{\alpha} \sigma \tau \rho о \varphi \circ \varsigma$

2 Vgl. Maurice Geerard, Clavis patrum Graecorum. II Ab Athanasio ad Chrysostomum (Corpus christianorum), Turnhout 1974, Nr. 2171-2309 und die Ergänzungen in Maurice Geerard/ Jacques Noret, Clavis patrum Graecorum. VI Supplementum (Corpus christianorum), Turnhout 1998, Nr. 2163-2329.

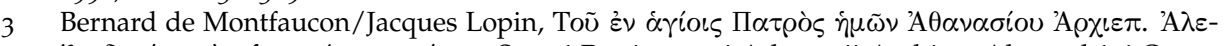
$\xi \alpha \nu \delta \rho \varepsilon i \alpha \varsigma \tau \grave{\alpha} \varepsilon \dot{v} \rho \iota \sigma x o ́ \mu \varepsilon \nu \alpha \tau \alpha \dot{\alpha} \tau \alpha$. Sancti Patris nostri Athanasii Archiep. Alexandrini Opera omnia quae exstant vel quae ejus nomine circumferuntur, Ad mss. codices Gallicanos, Vaticanos, \&c. necnon ad Commelinianas lectiones castigata, multis aucta: nova Interpretatione, Praefationibus, Notis, variis lectionibus illustrata: novà Sancti Doctoris vitâ, Onomastico, \& copiosissimis Indicibus locupletata. Opera \& studio monachorum ordinis S. Benedicti è congretatione Sancti Mauri. Tribus Tomis in folio Graece et Latine, Paris 1698. 
heute Stand der Forschung. Denn das Verdikt Montfaucons, daß ein unter dem Namen des Athanasius überlieferter Text unecht ist, hat in den meisten Fällen dazu geführt, daß diese überhaupt nicht mehr herangezogen, übersetzt oder untersucht wurden, ${ }^{4}$ weil sie ja zu unseren Kenntnissen über Athanasius, sein Leben und seine Theologie offensichtlich nichts beitragen könnten.

Nicht beachtet wird hierbei aber, daß auch pseudonyme Schriften sehr wohl unsere Kenntnisse erweitern können, und zwar über Autoren, deren Werke im Allgemeinen nur sehr schlecht überliefert oder die sonst überhaupt nicht mehr in ihren Werken greifbar sind, sowie darüber hinaus auch über die Kirchen- und Theologiegeschichte generell. Dies trifft gleichermaßen für die pseud-athanasianischen Schriften zu, die bisher noch nicht untersucht worden sind oder deren ursprünglicher Verfasser sich auch durch eine Untersuchung nicht mehr erheben läßt.

Im folgenden soll dies an einem solchen pseud-athanasianischen Text, der Disputatio contra Arium (CPG 2250), exemplarisch vorgeführt werden. ${ }^{5}$

\section{Hinführung zum Text}

Die Synode von Nizäa war nicht nur für die Zeitgenossen im Rückblick das Ereignis der Geschichte der Kirchen im konstantinischen Zeitalter: Sie war die erste »ökumenische « Synode ${ }^{6}$, auf ihr wurde erstmals ein für die gesamte Kirche auf dem Gebiet des Imperium Romanum (und sogar darüber hinaus) geltendes Bekenntnis formuliert und nicht zuletzt auch die wichtige liturgische Frage der Berechnung des Osterfestes geklärt.7

Waren die Beschlüsse der Synode im engeren Sinn, d.h. der Brief der Synode an die Kirche von Ägypten ${ }^{8}$, die theologische Erklärung ${ }^{9}$, die Canones ${ }^{10}$ und die (Unterschriften-)Liste der Teilnehmer ${ }^{11}$, noch schriftlich festgehalten worden, ${ }^{12}$

4 Eine Ausnahme bilden hier die heute Markell oder Apolinaris (und seinen Schülern) zugeschriebenen Schriften.

5 Dabei ist jedoch keine erschöpfende Interpretation des Textes beabsichtigt; vielmehr möchte ich den Text erst einmal nur grundsätzlich erschließen, wobei viele Fragen und Probleme nur sehr knapp angeschnitten werden können.

6 Vgl. Henry Chadwick, The Origin of the Title »Oecumenical Council«, JThS 23 (1972), 132-135; Annette von Stockhausen, Athanasius von Alexandrien. Epistula ad Afros. Einleitung, Kommentar und Übersetzung (PTS 56), Berlin/New York 2002, 102-105.

7 Vgl. Hanns Christof Brennecke, Nicäa I. Ökumenische Synode von 325, TRE 24 (1994), 429-441.

8 Dok. $25=$ Urk. 23.

9 Dok. $26=$ Urk. 24

10 Anon.Cyz., h.e. II 32; Ruf., h.e. X 6. Vgl. auch Thdt., h.e. I 8,18.

11 Heinrich Gelzer/Heinrich Hilgenfeld/Otto Cuntz (Hrsg.), Patrum Nicaenorum nomina Latine, Graece, Coptice, Syriace, Arabice, Armeniace (Bibliotheca scriptorum Graecorum et Romanorum Teubneriana), Stuttgart/Leipzig 1995.

12 Das heißt zumindest, insofern sie uns erhalten sind; es ist natürlich möglich, daß es noch weitere, heute verlorene Dokumente gab. 
so galt das nicht für die Verhandlungen selbst. ${ }^{13}$ In Ermangelung von Protokollen wurden daher immer wieder Berichte von an der Synode Anwesenden bzw. Beteiligten herangezogen: Der Brief Eusebs an seine Kirche ${ }^{14}$, der sich zwar nicht als offizielles Dokument der Synode gibt, aber vielleicht noch am ehesten als Bericht über den Ablauf der Verhandlungen herangezogen werden könnte, bietet jedoch eine sehr eigenwillige Interpretation des Verhandlungsverlaufes und ist keinesfalls als wie auch immer geartetes »Protokoll« der Verhandlungen anzusehen, sondern ist eine Apologie Eusebs für seine Zustimmung zur theologischen Erklärung der Synode. ${ }^{15}$ Ebenso sind die immer wieder einmal für die Rekonstruktion des Verlaufs der Synode herangezogenen Abschnitte aus der athanasianischen Epistula ad Afros zum einen auf jeden Fall um einiges, nämlich an die 40 Jahre, später anzusetzen, vor allem aber bieten sie zum anderen überhaupt keine Schilderung der Verhandlungen auf der nizänischen Synode, sondern verdanken sich einer nachträglichen Interpretation des Nizänums von Seiten des Athanasius. ${ }^{16}$

Zusammenfassend läßt sich feststellen, daß auf der Synode von Nizäa allem Anschein nach keine Protokolle angefertigt worden waren, an Hand derer sich der Verlauf der Diskussionen und Verhandlungen später hätte ablesen lassen können. Angesichts der im Laufe des 4. Jahrhunderts anwachsenden Bedeutung, die der Synode von Nizäa zugemessen wurde ${ }^{17}$ verwundert es daher nicht, daß ein Bedürfnis entstand, mehr über die Verhandlungen und Diskussionen zwischen »Orthodoxen« und »Arianern« auf der Synode von Nizäa zu erfahren,

13 Evangelos Chrysos, Konzilsakten und Konzilsprotokolle vom 4. bis 7. Jahrhundert, AHC 15 (1983), 30-40 hat anhand der überlieferten Protokolle des 5.-7. Jahrhunderts gezeigt, daß die Abfassung bzw. Nicht-Abfassung von Protokollen (im Gegensatz zu Akten) synodaler Verhandlungen in Abhängigkeit davon zu sehen ist, ob es sich um ein synodales Gerichtsverfahren handelte. Träfe des auch schon für das 4. Jahrhundert zu, so hätten für die Synode von Nizäa ebenfalls offizielle Protokolle angefertigt worden sein müssen, vgl. K. M. Girardet, Kaisergericht und Bischofsgericht. Studien zu den Anfängen des Donatistenstreites (313-315) und zum Prozeß des Athanasius von Alexandrien (328-346) (Antiquitas I 21), Bonn 1975, 43-51. Explizit von offiziellen Protokollen einer Synode wissen wir aber erst in Bezug auf eine antiochenische Synode im Jahr 327, die Asklepas von Gaza verurteilte (Dok 35.1 Brennecke/Heil/Stockhausen/Wintjes), in Bezug auf die Synode von Tyros (bzw. auf die von ihr eingesetzte Mareotis-Kommission, vgl. Ath., apol.sec. 5,4; 27,4; 28,4.6 f.; 31,1; 37,9; 83,4; h. Ar. 15,5; Socr., h.e. I 31,3.5; II 17,8) und in Bezug auf die Synode von Seleukia (Socr., h.e. II 39,7 f.: $\delta \eta \mu \omega \sigma i \omega \nu$ i $\pi \circ \mu \nu \eta \mu \alpha ́ \tau \omega \nu$, vgl. Soz., h.e. IV 22,28).

Zur älteren Diskussion der Frage, ob es Protokolle der Synode von Nizäa gab, vgl. Pierre Batiffol, Les sources de l'histoire du concile de Nicée, Échos d'Orient 24 (1925), 385-402; 30 (1927), 5-17, hier 386-388 (mit einer Zusammenfassung des älteren Forschungsstandes).

14 Dok. $24=$ Urk. 22.

15 Vgl. die beiden den Brief rahmenden Abschnitte Dok. 24,1.17.

16 Das habe ich in Stockhausen, Athanasius von Alexandrien. Epistula ad Afros, 219-238 gezeigt.

17 Ein Indikator für die wachsende Bedeutung der Synode ist die Neuberechnung der Zahl der auf der Synode anwesenden Bischöfe (allen zeitgenössischen Angaben zufolge an die 30o) auf die »biblische" Zahl 318 (der in Gen 14,14 genannten 318 Diener Abrahams), die zuerst im Kontext der Synode von Rimini/Seleukia auftaucht, vgl. Stockhausen, Athanasius von Alexandrien. Epistula ad Afros, 106-109. 
und daß daher bald Legenden über den Verlauf der Synode aufkamen und über die Disputationen, die auf ihr stattgefunden hatten.

Solche Legenden sind für uns zuerst in Rufins Fortsetzung der eusebianischen Kirchengeschichte greifbar, ${ }^{18}$ in der Rufin im Kontext der Synode von Nizäa über den Dialog mit einem arianischen Philosophen und über dessen anschließende Bekehrung berichtet. ${ }^{19}$

Aufgenommen wird diese Legende dann nicht nur in den Kirchengeschichten des Sokrates und des Sozomenos, ${ }^{20}$ sondern auch in der Kirchengeschichte des Anonymus von Kyzikos. ${ }^{21}$ Im Gegensatz zu seinen Vorgängern in der Kirchengeschichtsschreibung bringt dieser aber nun noch zusätzlich die Niederschrift eines langen Dialoges ${ }^{22}$ zwischen einer Gruppe »nizänischer « Bischöfe (Eustathius von Antiochia, Ossius von Cordoba, Leontius von Caesarea in Kappadokien, Eupsychios von Tyana, Euseb von Caesarea, Protogenes von Serdica und Macarius von Jerusalem) und einem arianischen Philosophen mit dem sprechenden sokratischen Namen Phaidon, die ihren Ausgangspunkt im Bibelvers Gen 1,26 nimmt.

Auch hier bekehrt sich am Ende der Philosoph Phaidon zum orthodoxen Christentum. Und dies ist eine auffällige Gemeinsamkeit, die wir im folgenden noch ein weiteres Mal antreffen werden: Am Ende bekennt sich der "arianische" Gesprächspartner immer zur »Orthodoxie«. Das ist zum einen sicherlich auch eine Folge des tief empfundenen Bedürfnisses nach ónóvoı $\alpha$ und des Horrors vor innerchristlicher Zwietracht, was beides ja überhaupt erst den Anlaß der nizänischen Synode gebildet hat, ${ }^{23}$ zum anderen verdankt es sich natürlich auch der theologischen Herkunft der jeweiligen Autoren.

Ein weiterer Protagonist solcher auf der Synode von Nizäa verankerter Dialoge ist nun der damalige Diakon und spätere Bischof Athanasius von Alexandrien, der seinen damaligen Bischof Alexander wohl auf die Synode begleitet hatte. ${ }^{24}$

Den Anlaß dafür, daß er diese Rolle in Dialogen immer wieder einnahm, bot Athanasius wahrscheinlich sogar selbst, wenn er in Apologia secunda 6,2 über seine Aktivitäten auf der Synode schreibt:

18 Zur Frage, inwieweit Rufin dabei auf die sonst verlorene Kirchengeschichte des Gelasius von Caesarea zurückgreift, vgl. zusammenfassend Philip R. Amidon, The church history of Rufinus of Aquileia. Books 10 and 11, Oxford/New York 1997, XIII-XVII.

19 Ruf., h.e. X 3. Vgl. dazu M. Jugie, La dispute des philosophes paiens avec les pères de Nicée, Échos d'Orient 24 (1925), 403-410; Françoise Thelamon, Païens et chrétiens au IVe siècle. L'apport de $l^{\prime}$ «Histoire ecclésiastique » de Rufin d'Aquilée, Paris 1981, 430-435. Daß eine natürliche Affinität von Arianismus und Philosophie besteht, ist dabei natürlich polemischer Topos.

20 Vgl. Sokr., h.e. I 8; Soz., h.e. I 18.

21 Anon.Cyz., h.e. II 13.

22 Anon.Cyz., h.e. II 14-24 (50,6-82,26 Hansen).

23 Vgl. Brennecke, Nicäa I, v.a. $429 \mathrm{f}$.

24 Zur Frage der Anwesenheit des Athanasius auf der Synode von Nizäa vgl. Stockhausen, Athanasius von Alexandrien. Epistula ad Afros, 219 m. Anm. 1. 
Da Alexander wegen seiner Frömmigkeit in Christu den Frevler (sc. Arius) nicht aufnahm, richteten sie (die Arianer) gegen Athanasius, der damals Diakon war, ihre Missgunst. Denn in ihrer Umtriebigkeit vernahmen sie, daß er häufig mit Alexander zusammen war und bei ihm in Ehren stand. Als sie mit ihm und seiner Frömmigkeit in Christus auf der in Nicaea versammelten Synode Bekanntschaft machten - und zwar dadurch, daß er offen gegen die Häresie der Ariomaniten auftrat -, wurde ihr Hass noch größer. ${ }^{25}$

Natürlich hat dann in der Folgezeit auch sein Nimbus als "Säule der Orthodoxie $\ll^{26}$ und als wegen seiner Konfrontationen mit Arianern aller Coleur fünfmal Verbannter $^{27}$ sein übriges dazu getan, daß Athanasius sich als Protagonist antiarianischer Dialoge geradezu anbot.

So überrascht es nicht, daß unter seinem Namen mehrere Disputationen mit Arius bzw. mit anderen Nicht-Nizänern und dann ganz allgemein im Kontext der trinitätstheologischen Streitigkeiten überliefert sind. ${ }^{28} \mathrm{Zu}$ nennen sind hier z.B. der lateinisch verfasste Contra Arianos dialogus. ${ }^{29}$ Aber auch die unter dem Namen des Athanasius überlieferten Dialoge, die ihn jedoch nicht zugleich als Protagonisten auftreten lassen, wie die von Christoph Bizer untersuchten Dialoge De sancta trinitate $I-V^{30}$ und die Dialogi contra Macedonianos I und $I^{31}$ gehören hier dazu. ${ }^{32}$

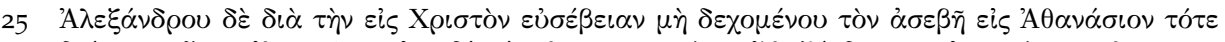

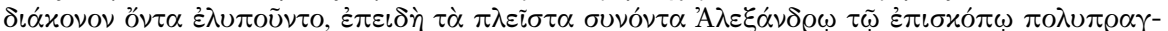

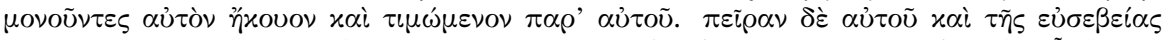

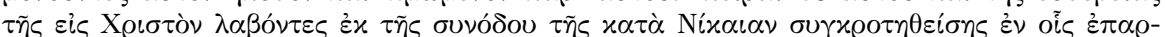

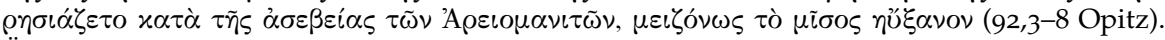
Übersetzung Werner Portmann, Athanasius, Zwei Schriften gegen die Arianer. Verteidigungsschrift gegen die Arianer / Geschichte der Arianer (BGL 65), Stuttgart 2006, 84. Weitere Stellen führt Annick Martin, Athanase d'Alexandrie et l'eglise d'Egypte au IV siècle (328-373), Rom 1996, 321 Anm. 1 und 2 an.

26 Vgl. Stellen wie Greg.Naz., or. 21,26 oder Paul.Em., hom. 2,6 (ACO I 1,4, 14,14 f.).

27 Zur Wahrnehmung des Athanasius als Confessor vgl. Äußerungen wie die des Cyr., apol.Thds. 29 (ACO I 1,3, 89,17-20).

28 Daß der Protagonist eines Dialogs im Laufe der Überlieferung auch zu dessen Autor wird, ist ein auch sonst zu beobachtendes Phänomen, vgl. Gerhard Ficker, Studien zu Vigilius von Thapsus, Leipzig 1897, 34.39-41 (s. auch die nachfolgende Anm.) und Christoph Bizer, Studien zu pseudathanasianischen Dialogen der Orthodoxos und Aëtios, Diss., Bonn: theol., 1970, 67.

29 Der Text liegt in einer längeren Rezension vor, die Vigilius von Thapsus zum Autor hat (CPL 807 [PL 52,179-238]): »Contra Arianos, Sabellianos, Photinianos dialogus«. Außerdem ist er in einer von einem anonymen Exzerptor angefertigten kürzeren Rezension unter dem Titel "Contra Arianos Athanasio Ario et Probo judice interlocutoribus« bzw. »Disputatio Athanasii cum Ario coram Probo judice« überliefert (CPL 812 [PL 52,155-18o, vgl. PG 28,1439 f.]). Beide Fassungen werden in Teilen der Überlieferung Athanasius als Autor zugeschrieben, vgl. dazu insgesamt Ficker, Studien zu Vigilius von Thapsus, 25-42.

30 CPG 2284, vgl. Bizer, Studien.

31 CPG 2285.

32 Zugleich zeugen all diese Dialoge ganz allgemein sehr eindrücklich von der Rezeption und vom neuerlichen Aufblühen der altehrwürdigen Gattung Dialog ab der zweiten Hälfte des 4. Jahrhunderts, vgl. B. R. Voss, Der Dialog in der frühchristlichen Literatur, München 1970, $33^{8}-341 \cdot 347-352$. 


\section{Die Disputatio contra Arium}

\section{Die Überlieferung des Textes}

Da die Disputatio contra Arium ${ }^{33}$ bisher in keiner kritischen Edition vorliegt, aber auch aus inhaltlichen Gründen, für die die Überlieferungszusammenhänge wichtig sind, soll zunächst einiges zur Überlieferung der Disputatio contra Arium vorangeschickt werden:

Die Disputatio contra Arium ist im Rahmen der sogenannten $x$-Sammlung, außerhalb des Kontextes der großen Athanasius-Sammlungen auch in einigen weiteren, meist späten (Sammel-)Handschriften ${ }^{34}$, in einer lateinischen ${ }^{35}$ und (allerdings nicht ganz vollständig) in einer armenischen Übersetzung ${ }^{36}$ überliefert. Eine kurze Passage, das Bekenntnis des Athanasius in Kap. $5^{37}$, ist außerdem noch als singuläres Stück in einem Codex der Pariser Nationalbibliothek überliefert. $3^{8}$

Über die direkte Überlieferung hinausgehend lassen sich auch geringe Spuren einer Sekundärüberlieferung der Disputatio contra Arium feststellen. 39

33 CPG 2250; der Text ist in PG 28,440-501 abgedruckt. Eine kritische Edition liegt nicht vor Überhaupt erwähnen den Text m.W. nur Voss, Dialog, 333 Anm. 50 und (in überlieferungsgeschichtlichem Zusammenhang) Robert Pierce Casey, Armenian Manuscripts of St. Athanasius of Alexandria, HThR 25 (1932), 43-59, hier 46 f., Charles Renoux, Athanase d'Alexandrie dans le florilège arménien du Manuscrit Galata 54 (Deuxième Partie), Handes Amsorya 103 (1989), 7-27, hier $25 \mathrm{f}$. und Bizer, Studien, 41-70 (passim).

34 Bekannt sind mir bisher die Handschriften Codex Parisinus gr. 854 (s. XIII), 1327 (a. 1562) und Parisinus Suppl. gr. 168 (s. XV); Bizer, Studien, 50-53.65 f. führt außerdem die Handschriften Codex Oxoniensis Laudeanus gr. 26 (s. XVI), Codex Caroliruhensis gr. 393 (s. XV) und Codex Vaticanus gr. 402 (a. 1383) an.

35 Bekannt ist mir bisher die im Codex Londinensis Regius 5 F. ii (a. 1446) unter dem Titel »De unitate substantiae deitatis dialogus « überlieferte Übersetzung. Wahrscheinlich ist sie aber erst im Kontext des Konzils von Florenz entstanden.

36 Vgl. Casey, Armenian Manuscripts of St. Athanasius of Alexandria, Renoux, Athanase d'Alexandrie und oben S. 47 f. im Beitrag von Anahit Avagyan.

37 PG 28,441D-444A.

38 Cod. Parisinus Regius 2280, s. XIV, f. 13. Die heutige Sigle der Handschrift und damit dann auch den Überlieferungszusammenhang habe ich noch nicht herausfinden können, da der Handschriftenkatalog von Henri Omont, Inventaire sommaire des manuscrits grecs de la Bibliothèque nationale I-IV, Paris 1886/1888/1898 in die Irre führt. Zu finden ist das Stück aus dem Pariser Codex unter den Fragmenta varia (CPG 2165.23) in PG 26,1321D-1323A; zuerst ediert ist es in Bernard de Montfaucon, Sancti patris Nostri Athanasii Opuscula secundis curis reperta et antehac inedita (Collectio nova patrum et scriptorum graecorum, Eusebii Caesariensis, Athanasii, \& Cosmae Aegyptii), Paris 1706, 106.

39 Zitate konnte ich bisher nur im Kontext der hesychastischen Kontroverse bei Gregorios Akindynos, Refutatio magna, vgl. den Stellenindex in Juan Nadal Cañellas, Gregorii Acindyni refutationes duae operis Gregorii Palamae cui titulus Dialogus inter orthodoxum et Barlaamitam (CChr.SG 31), Turnhout 1995, und Nikephoros Gregoras, Historia Romana, vgl. Immanuel Bekker, Nicephori Gregorae Byzantina historia III (CSHB), Bonnae 1855, und im Kontext des Konzils von Florenz (pneumatologische Frage), vgl. Sessio 4 (317,17-27 Gill) und Sessio 5 $(335,30-336,2$ Gill), ausmachen. 
Bei der $x$-Sammlung ${ }^{40}$ handelt es sich um eine eigenständige, fest umrissene Zusammenstellung von Schriften des Athanasius, deren besonderes Kennzeichen es ist, daß ihr ein Inhaltsverzeichnis und Exzerpte aus einem Brief des Photios an seinen Bruder Tarasios über die Schriften des Athanasius vorangehen ${ }^{41}$ und daß sie außerdem in ihrer Zusammenstellung (im Gegensatz vor allem zur $y$-Sammlung, aber auch zur $b$-Tradition $)^{42}$ sehr homogen überliefert ist.

Sie enthält die folgenden 21 athanasianischen und pseud-athanasianischen ${ }^{43}$ Schriften: Oratio contra gentes (CPG 2090), Oratio de incarnatione verbi (CPG (2091), †Disputatio contra Arium (CPG 2250), Epistula ad episcopos Aegypti et Libyae (CPG 2092), Orationes contra Arianos I-III (CPG 2093), +De incarnatione et contra Arianos (CPG 2806), Epistula encyclica (CPG 2124), Epistulae ad Serapionem I-II (CPG 2094), †Epistula catholica (CPG 2241), +Refutatio hypocriseos Meletii et Eusebii (CPG 2242), Epistula ad Epictetum (CPG 2095), +Contra Apolinarem II-I (CPG 2231), In illud: qui dixerit verbum in filium (CPG 2096), + De passione et cruce domini (CPG 2247), Epistula ad Marcellinum (CPG 2097), De virginitate (CPG 2248) und tTestimonia e scriptura (CPG 2240).

Wie an dieser Aufzählung ersichtlich ist, steht die Disputatio contra Arium zwar nicht ganz zu Beginn der Sammlung. Indem sie aber nach (den die arianische Frage nicht behandelnden) Schriften Contra gentes und De incarnatione zu stehen kommt, fungiert sie gewissermaßen als Einleitung zu den »antiarianischen « Schriften des Athanasius, die in dieser Sammlung zusammengefaßt

40 Die Sammlung wird von Opitz nach einem der Hauptüberlieferungsträger auch als WSammlung bezeichnet, vgl. Hans-Georg Opitz, Untersuchungen zur Überlieferung der Schriften des Athanasius (AKG 23), Berlin/Leipzig 1935, 181-188. Zur Sammlung sind außerdem Hanns Christof Brennecke/Uta Heil/Annette von Stockhausen, Athanasius Werke II 8. Die »Apologien «, Berlin/New York 2006, xiv f. und die entsprechenden Einträge in Karin Metzler/Dirk Uwe Hansen/Kyriakos Savvidis, Athanasius Werke. Band I/Teil 1: Die Dogmatischen Schriften. Lfg. I Epistula ad episcopos Aegypti et Libyae, Berlin/New York 1996, Karin Metzler/Dirk Uwe Hansen/Kyriakos Savvidis, Athanasius Werke. Band I/Teil 1: Die Dogmatischen Schriften. Lfg. 2 Orationes I et II contra Arianos, Berlin/New York 1998 und Karin Metzler/Kyriakos Savvidis (Hrsg.), Athanasius Werke. Band I/Teil 1: Die Dogmatischen Schriften. Lfg. 3 Oratio III contra Arianos, Berlin/New York 2000 zu vergleichen.

41 Der Brief ist ediert in Opitz, Untersuchungen, 213. Daß Pinax und Brief Teil der Sammlung sind, läßt sich sehr schön an Codex Athous Vatopedi 7 (W), in dem Brief und Pinax erst auf f. 99a ff., zu Beginn des zweiten Teiles der Handschrift zu stehen kommen, und an Codex Marcianus gr. Z 49 (M) erkennen, bei dem der Pinax auf f. 2a-b nur die zur $x$-Sammlung gehörenden Stücke anführt, nicht aber die Stücke aus anderen Sammlungen, die der Codex auch enthält; wie eine Autopsie der Handschrift am 12.10.2002 ergeben hat, sind die Angaben in Opitz, Untersuchungen, 21 nicht korrekt: Der Pinax auf f. 1b, der ebenfalls nur einen Teil der Schriften enthält, stammt nicht von einer älteren, sondern von einer jüngeren, wahrscheinlich dem 15. Jh. angehörenden Hand (der Codex selbst ist wohl ins späte 13. Jh. zu datieren), der Pinax auf f. 2a-b enthält nur die Titel Nr. 1-21 ( $=x$-Sammlung) und nicht alle im Codex unter dem Namen des Athanasius überlieferten Schriften.

$42 \mathrm{Zu}$ den beiden Überlieferungszusammenhängen knapp Brennecke/Heil/Stockhausen, Athanasius Werke II $8, x v-x v i i$ mit Literatur.

43 Markiert mit + . 
sind, ${ }^{44}$ insofern die Disputatio contra Arianos dadurch, daß sie eine Diskussion zwischen Athanasius und Arius auf der Synode von Nizäa selbst wiedergibt, die thematisch und chronologisch nach der Synode von Nizäa einzuordnenden Schriften des Athanasius an diese zurückbindet und die Hintergründe des Streites erhellt.

Nun ist die Zusammenstellung des Schriftenkorpus der $x$-Sammlung wegen der Inklusion des Photios-Briefes frühestens im späten 9. Jahrhundert anzusetzen. Allerdings könnte gerade der Photios-Brief selbst eine Zeuge für eine ältere und weniger umfangreiche Sammlung antiarianischer Schriften des Athanasius sein:

Im Brief an Tarasios und in cod. 140 der Bibliothek schreibt Photios nämlich von einer Sammlung von Werken des Athanasius, der $\varkappa \alpha \tau \grave{\alpha}$ 'A

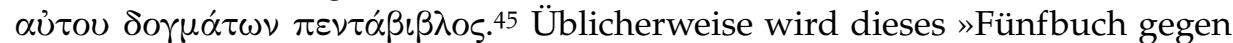
Arius und seine Lehren " auf die Epistula ad episcopos Aegypti et Libyae, die drei Arianerreden und die pseud-athanasianische vierte Arianerrede bezogen, ${ }^{46}$ weil in den Handschriften der y-Sammlung47 die Epistula ad episcopos Aegypti et Libyae, die drei Arianerreden und die pseud-athanasianische vierte Arianerrede als $\varkappa \alpha \tau \grave{\alpha}$ 'A $\rho \varepsilon \iota \alpha \nu \tilde{\omega} \nu \lambda o_{\gamma}{ }^{\prime} \alpha^{\prime}-\varepsilon^{\prime}$ gezählt werden..$^{48}$ Demgegenüber werden in der $x$ Sammlung nun gerade nur die drei echten Arianerreden durchnummeriert, nicht aber die Epistula ad episcopos Aegypti et Libyae in diese Zählung einbezogen. ${ }^{49}$

Wäre nun die Identifikation der Pentabiblos mit der Zusammenstellung von Epistula ad episcopos Aegypti et Libyae, den drei authentischen und der pseudonymen Arianerrede richtig, dann würde nun allerdings der Inhalt der $x$-Sammlung nicht zu den im Brief genannten Schriften passen, da ja die vierte Arianerrede nie Teil dieser Sammlung war. Dies könnte natürlich auf einen Fehler dessen zurückzuführen sein, der die $x$-Sammlung zusammen- und ihr den Brief vorangestellt hat. ${ }^{\circ}$ Außerdem muß zumindest fraglich bleiben, ob denn Photios

44 Also zumindest zu den Schriften Epistula ad episcopos Aegypti et Libyae, den drei Arianerreden, De incarnatione et contra Arianos und der Epistula encyclica.

45 Opitz, Untersuchungen, 213,12 und Immanuel Bekker, Photii Bibliotheca I, Berlin 1824, 98a.

46 Vgl. z.B. Metzler/Hansen/Savvidis, Athanasius Werke I/2, $71 \mathrm{f}$.

47 Nur in dieser Sammlung ist die vierte Arianerrede nämlich überhaupt überliefert.

48 Vgl. Markus Vinzent, Pseudo-Athanasius, contra Arianos IV. Eine Schrift gegen Asterius von Kappadokien, Eusebius von Cäsarea, Markell von Ankyra und Photin von Sirmium (SVigChr 36), Leiden [u.a.] 1996 und Anton Stegmann, Die pseudoathanasianische »IVte Rede gegen

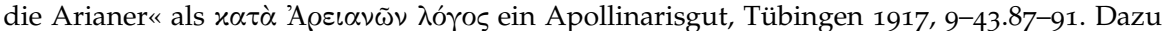
werden in Teilen der Sekundärüberlieferung die Epistula ad episcopos Aegypti et Libyae und

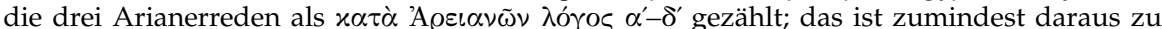
schließen, daß die dritte Arianerrede z.B. Florilegium Athanasium, fr. 99 (36 Schwartz) oder Justn., monoph. $126(28,15$ f. Schwartz) als vierte Rede gezählt wird.

49 Eine Ausnahme bilden Codex Patmiacus A 3 (U) und Codex Marcianus gr. $50(\mathrm{~N})$, die die Texte wie die Handschriften der $y$-Sammlung durchnummerieren; allerdings hat Karin Metzler, Kontamination in der Athanasius-Überlieferung, REByz 48 (1990), 213-232 gezeigt, daß diese beiden Handschriften von der $y$-Sammlung her kontaminiert sind.

50 Zumal im Brief über die Angaben in cod. 140 hinausgehend noch weitere Schriften genannt werden, die ebenfalls nie Teil der $x$-Sammlung waren, vgl. Opitz, Untersuchungen, 213,18-22 und cod. 32 sowie cod. 139 . 
überhaupt die vierte Arianerrede zu den echten Athanasiana gezählt hat; in den Werken des Photios wird sie sonst wenigstens nicht erwähnt und auch in der übrigen Sekundärüberlieferung wird die Rede so gut wie nie als Athanasianum wahrgenommen. ${ }^{51}$

Ich möchte daher eine andere Hypothese aufstellen und vorschlagen, die

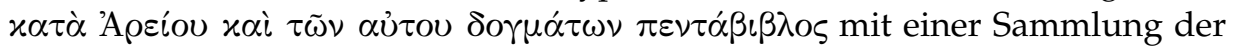
Schriften Disputatio contra Arium, Epistula ad episcopos Aegypti et Libyae, Arianerreden I-III zu identifizieren. ${ }^{2}$

\section{Der Titel der Schrift}

Der Titel der Schrift lautet in der überwiegenden Mehrzahl der Handschrif-

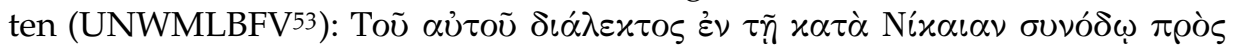
'A $\rho \varepsilon \iota$, also: »Desselben Gespräch mit Arius auf der Synode von Nizäa«.

Nur Codex Vatopedi 5/6 (K) ${ }^{54}$ bietet den durch Montfaucon und dann Migne kanonisch gewordenen Titel Disputatio contra Arium, da er in ihr lautet: Toũ हैv

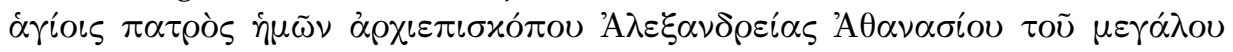

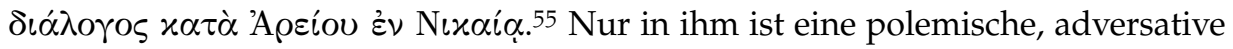
Komponente enthalten, die im Text des Dialogs selbst - bei aller theologischer Kontroverse - keine rechte Basis hat. ${ }^{56}$ Dieser Titel verdankt sich eher der allgemeinen antihäretisch ausgerichteten Rezeption der arianischen Kontroverse als einer aktuellen Lektüre des Dialoges. 57

Noch interessanter ist jedoch die Titelvariante, die vom (freilich späten) Codex Laurentianus Riccardianus $4(\mathrm{Q})^{5^{8}}$ und von der armenischen Überset-

51 Vgl. dazu Vinzent, Pseudo-Athanasius, contra Arianos IV, $1 \mathrm{f}$.

52 Ich werde auf diese Hypothese am Schluß noch einmal zurückkommen, wenn die Frage der Autorschaft (auch diese in Form einer Hypothese) geklärt ist, vgl. unten S. 152.

53 Zur Auflösung der Siglen vgl. Brennecke/Heil/Stockhausen, Athanasius Werke II 8, cxxvi, Metzler/Hansen/Savvidis, Athanasius Werke I/1, 36 oder Opitz, Untersuchungen, IX.

54 Der Codex Vatopedi $5 / 6$ ist zusätzlich dadurch auffällig, als die Disputatio contra Arium in ihm nicht im Kontext der $x$-Sammlung (in Codex Vatopedi 5), sondern im Kontext von Schriften, die der $y$-Sammlung angehören (die Reihenfolge der Schriften der $y$-Sammlung ist in $\mathrm{K}$ allerdings gestört), in Codex Vatopedi 6 überliefert ist; vgl. Opitz, Untersuchungen, 38-40. Hier kann erst eine Kollation des Textes zu weiteren Erkenntnissen führen, z.B. ob K etwa auch einen anderen Text als die übrigen Handschriften überliefert.

55 In der Refutatio magna des Gregorios Akyndinos finden sich beide Titelvarianten, vgl. Cañellas, Gregorii Acindyni refutationes duae, Index. Er und Nikephoros Gregoras zitieren den Text auch

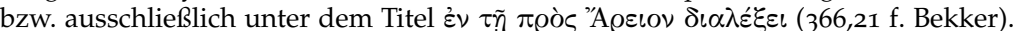

56 Aus Gründen der Praktikabilität bleibe ich dennoch bei dem eingeführten lateinischen Titel Disputatio contra Arium.

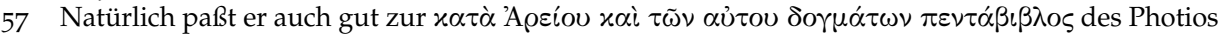
(s. oben).

58 Der Codex Laurentianus Riccardianus 4 (Q) ist zwar eine Abschrift des Codex Oxoniensis Holkham gr. 28 ( $\mathrm{h}=74)$, doch leider läßt sich dem Katalog (vgl. Brennecke/Heil/Stockhausen, Athanasius Werke II 8, lviii mit allen Angaben) nicht entnehmen, wie der Titel der Disputatio contra Arium in ihm lautet. Der Codex Oxoniensis Holkham gr. $28(\mathrm{~h}=74)$ selbst ist wiederum eine Abschrift des Codex Londinensis Burneianus gr. 46 (L), der nicht die Langform des Titels 


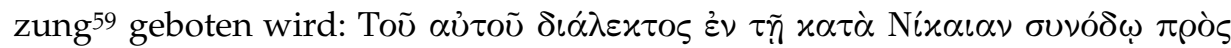

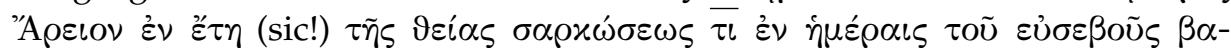

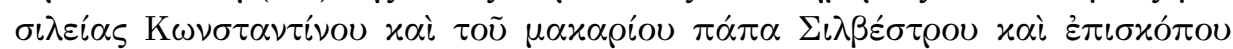

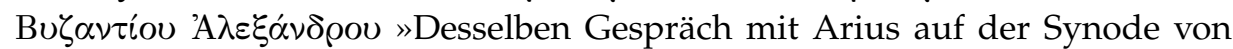
Nizäa im Jahr der göttlichen Fleischwerdung 310, in den Tagen des frommen Kaisers Konstantin und des seligen Papstes Silvester und des Bischofs von Byzanz, Alexander .

Auffällig ist an diesem Titel zunächst vor allem die Datierung der Synode von Nizäa in das Jahr anno domini 310, da hier auf den ersten Blick ein offensichtlicher Fehler vorzuliegen scheint.

Es ist jedoch anzunehmen, daß die Jahreszahl nicht fehlerhaft vom Verfasser dieses Titel angegeben worden, sondern nur fehlerhaft überliefert ist, daß also eine Korruptel vorliegt: ${ }^{60}$ Es erscheint naheliegend, $\overline{\tau \iota} \mathrm{zu} \overline{\tau \iota \varepsilon} \mathrm{zu}$ konjizieren, da der Text nach der Jahreszahl mit $\grave{\varepsilon} \nu$ fortfährt, somit also nur der Buchstabe $\varepsilon$ ausgefallen wäre. Geht man von dieser Konjektur aus, ergibt sich eine Datierung ins Jahr 315 anno domini, welche gemäß der alexandrinischen Weltära, ${ }^{61}$ die die Inkarnation ins Jahr 9 n.Chr. setzt, zur richtigen zeitlichen Einordnung der Synode von Nizäa führen würde. ${ }^{62}$

Die Angabe des Datums der Synode von Nizäa nach der alexandrinischen Weltära hat zur Konsequenz, daß zumindest dieser Titel, wenn nicht die gesamte Disputatio contra Arium, mit einiger Wahrscheinlichkeit in Alexandrien zu verorten ist. $^{63}$

hat, vgl. Opitz, Untersuchungen, $23-25$ und Metzler/Hansen/Savvidis, Athanasius Werke I/1, $11 \mathrm{f}$.

Ebenfalls eine Abschrift von $\mathrm{h}=74$ ist der sog. Codex Felkckmanni $2=$ Codex Genevensis gr. 29 tom. I, der die Basis für die Editio Commeliniana bildete. Vgl. Brennecke/Heil/Stockhausen, Athanasius Werke II 8, lviii $\mathrm{f}$.

59 Vgl. oben Anm. 36.

60 Die armenische Übersetzung überliefert zwar ebenfalls die falsche Jahreszahl 310, vgl. Casey, Armenian Manuscripts of St. Athanasius of Alexandria, $46 \mathrm{f}$. das kann jedoch darauf zurückzuführen sein, daß die Übersetzung erst angefertigt wurde, als der griechische Text bereits fehlerhaft überliefert wurde. Leider steht nicht fest, wann die armenische Übersetzung entstanden ist.

61 Die alexandrinische Weltära ist zu Beginn des 5. Jahrhunderts unter Kyrill von Alexandriens Vorgänger Theophilos von Annianos in Alexandrien konzipiert worden, vgl. Georges Declercq, Anno Domini. The origins of the Christian era, Turnhout 2000, 29-34 und Alden A. Mosshammer, The Easter computus and the origins of the Christian era (Oxford early Christian studies), Oxford 2008, 362-368.

62 Die Zählung der Jahre der alexandrinischen Weltära verschiebt sich nämlich wegen einer Korrektur bei der Datierung des Todesjahres Jesu noch um ein Jahr, vgl. Mosshammer, Easter computus, 363.371 .

63 Es bleibt natürlich eine Unsicherheit, da die alexandrinische Weltära später auch über Alexandrien hinaus Verbreitung fand und bis ins 9. Jahrhundert in der Kirche üblich war. Der erste Beleg für ihre Verwendung liegt nach Declercq, Anno Domini, 34 zwar erst bei Kyrill von Skythopolis vor, aber zumindest für Alexandrien ist davon auszugehen, daß sie schon im 5. Jahrhundert auch Verwendung fand. Wenn sich also der Text auf Grund anderer Überlegungen ins Alexandrien des 5. Jahrhunderts datieren läßt, dann könnte die Verwendung der alexandrinischen Weltära als zusätzlicher Anhaltspunkt dienen. Vgl. unten S. 150. 
Stellt man nun die Langform des Titels mit ihren genauen Datums-Angaben ${ }^{64}$ der Fassung des Titels ohne diese Datierung abwägend gegenüber, so kann man zu dem Schluß kommen, daß die Langform vielleicht sogar der ursprünglichere Titel ist: Denn zum einen war die Datierung spätestens, als die alexandrinische Weltära von der byzantinische Weltära im 9. Jahrhundert abgelöst wurde, nicht mehr richtig und wäre kaum so von einem Kopisten eingefügt worden. ${ }^{65}$ Zum anderen stellt die Langform des Titels, zumal mit der falschen Jahresangabe, die lectio difficilior dar, da die Verkürzung des Titels im Laufe der Überlieferung wahrscheinlicher ist als die Hinzufügung weiterer Angaben. ${ }^{66}$

\section{Der Aufbau des Dialoges}

Der Dialog setzt - ganz im Stil eines sokratischen Dialogs ${ }^{67}$ - mit einer Straßenszene ein:

Athanasius, der Ich-Erzähler, ist zusammen mit einigen seiner Kleriker auf dem Weg zu einem Treffen mit weiteren, aber ebenfalls nicht näher identifizierten Gleichgesinnten. Auch der Ort der Szene wird nicht benannt, ebenso wenig das Ziel der »Wanderung «. Die Gruppe bewegt sich langsam vorwärts - »wegen der Schwäche des Körpers", wie Athanasius sagt ${ }^{68}$ - und während des Gehens wird Athanasius von einem seiner Begleiter nach der Bedeutung des Verses Joh 14,28 und damit nach der Bedeutung eines der loci classici des arianischen Streites gefragt. ${ }^{69}$

Damit ist im Grunde das Thema des gesamten Dialoges nach nur wenigen Zeilen umrissen: Es geht um die biblische Begründung arianischer Theologie.

Athanasius aber möchte die mit der Frage nach der Bedeutung von Joh 14,28 angeschnittene Diskussion zunächst auf einen anderen, vermeintlich günstigeren Zeitpunkt nach ihrer Rückkehr verschieben, muß dann aber "plötzlich « an

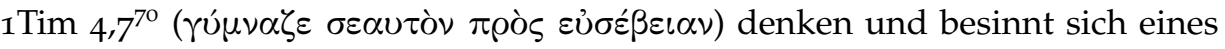
Besseren. ${ }^{71}$

64 Zur Jahresangabe anno domini kommt ja noch die Datierung nach den Bischöfen von Rom (Silvester) und Byzanz (Alexander).

65 Alle Handschriften, die die Disputatio contra Arium überliefern, sind ja später.

66 Es bleibt natürlich Aufgabe weiterer Untersuchungen zu klären, warum zumindest die indirekte Vorlage des Codex Laurentianus Riccardianus $4(\mathrm{Q})$, der Codex Londinensis Burneianus gr. 46 (L), nicht die Langform des Titels bietet, vgl. oben Anm. 58.

67 Vgl. Hermann Gundert, Der platonische Dialog (Bibliothek der klassischen Altertumswissenschaften. Neue Folge 2,26), Heidelberg 1968 und Hermann Gundert, Dialog und Dialektik. Zur Struktur des platonischen Dialogs (Studien zur antiken Philosophie 1), Amsterdam 1971; zu den Örtlichkeiten des sokratischen Dialogs auch Dietram Müller, Raum und Gespräch: Ortssymbolik in den Dialogen Platons, Hermes 116 (1988), 387-409.

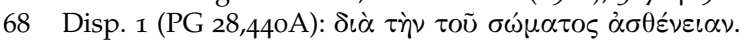

69 Disp. 1 (PG 28,440A).

70 Mit dem Zitat 1 Tim 4,7 ist bereits ein weiterer wichtiger Aspekt angeschnitten, der bei der Einordnung des Textes noch einmal in den Blick genommen werden muß: der Aspekt der $\gamma v \mu \nu \alpha \sigma i \alpha$, der »Übung «, vgl. unten S. 151.

71 Disp. 1 (PG 28,440B). 
Offensichtlich wird das Ziel des Weges aber noch erreicht, bevor Athanasius die gestellte Frage nach der Bedeutung von Joh 14,28 beantworten könnte; zumindest hören wir erst einmal nichts mehr davon, daß Athanasius die Frage nun doch noch beantwortet hätte. Denn nach ihrer Ankunft am Ziel des Weges begrüßt sich die Gruppe der Wanderer und die derer, die sich schon vor Ort

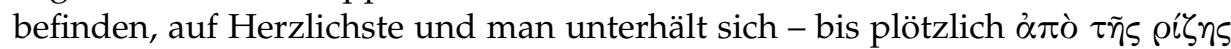

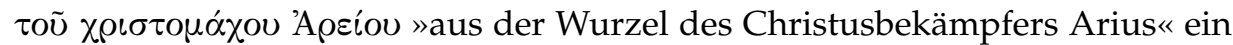
Inoíov $\alpha \nu \theta \rho \omega \pi$ ó $\mu \rho \rho \circ \nu$ ein "menschengestaltiges Tier « dazukommt und vor der versammelten Mannschaft eine Belehrung über den rechten Glauben fordert, und natürlich auch selbst seine Meinung darlegen möchte. ${ }^{22}$ Damit kommt die zunächst aufgeschobene Diskussion doch noch in Gang.

Athanasius fordert nun Arius auf (und folgt dabei ganz den Regeln der Rhetorik), seine $\pi \rho o ́ \tau \alpha \sigma \iota \varsigma$ vorzubringen, $\mathrm{d}$.h. seine These aufzustellen, ${ }^{73}$ womit der szenische Eingangsteil abgeschlossen wird und der dialogische Teil, die Disputation im eigentlichen Sinne, einsetzt.

Arius $^{74}$ schlägt daraufhin seinerseits vor, daß jeder der beiden Disputatenten in Kurzform ( $\dot{\varepsilon} \varepsilon \dot{\varepsilon} \pi \tau \circ \mu \tilde{n})$ darlegt, was er über »Gott, Christus und den Hl. Geist« denkt, damit dies als Grundlage der Diskussion für alle Beteiligten von vornherein klar sei. ${ }^{75}$ Hierauf formulieren sowohl Athanasius als auch Arius ein Bekenntnis. Athanasius formuliert:

Ich glaube an Gott, den Vater, den Allherrscher, der immer Gott Vater ist, und an den Gott Logos, den einziggeborenen Sohn Gottes, daß er mit seinem eigenen Vater zusammen existiert, und daß der Sohn aus dem Wesen des Gottes und Vaters ist, und daß der Sohn dem Vater gleich ist, und daß der Sohn Gott gleich würdig ist, und daß er zusammen mit seinem Vater überall in der Gottheit ist, und daß er alles in seinem Wesen umfasst, und daß der Sohn Gottes von niemandem umfasst wird, wie auch der Gott, sein Vater; und an den Hl. Geist; daß er vom Wesen des Vaters ist und der Hl. Geist gleichewig mit dem Vater und dem Sohn ist. Vom Logos sage ich aber, daß er im Fleisch geworden ist. ${ }^{6}$

Sieht man sich dieses Bekenntnis des Athanasius an, fällt auf den ersten Blick natürlich eines auf: Es handelt sich um ein Bekenntnis des »Nizäners" Athanasius, der noch dazu später nichts mehr anderes als das Nizänum gelten lassen wollte, das von ihm, dem Titel der Schrift nach, auf der Synode von Nizäa formuliert wurde - und das nichts mit dem Nizänum zu tun hat. Vor allem fällt

72 Disp. 3 (PG 28,441B).

73 Disp. 3 (PG 28,441C).

74 Nun, ab disp. 4 (PG 28,441C), werden die einzelnen Äußerungen der Dialogpartner auch jeweils durch Voranstellung ihres Namens gekennzeichnet.

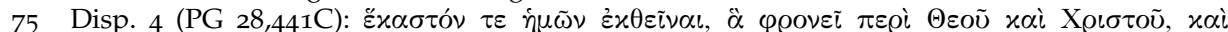

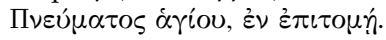

76 Disp. 5 (PG 28,441D-444A); der griechische Text folgt in der tabellarischen Übersicht unten. 
auf, daß der Begriff, der für Athanasius zum Schibbolet für Orthodoxie und zum

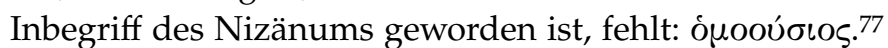

Das Bekenntnis des Arius, das folgt, ist zwar durch typisch "arianische« Theologumena gekennzeichnet, folgt aber ebenfalls nicht ganz den arianischen Sprachmustern:

Auch ich glaube an den einen ewigen Gott, und an seinen Sohn, den vor Ewigkeiten Gott gründete, und zum Sohn machte. Und alles, was der Sohn hat, hatte er nicht und empfing es von Gott. Und daß der Sohn nicht gleich ist und dem Vater nicht gleich würdig, sondern es fehlt ihm viel von der Würde Gottes, wie einem geschaffenen Ding fehlt es ihm an der Macht Gottes. Und an den Hl. Geist, der vom Sohn geworden ist. Siehe, auch ich denke so. ${ }^{78}$

Es ist ziemlich offensichtlich, daß das Bekenntnis des Arius nicht als eigenständiger Text entworfen ist, sondern von dem Bekenntnis des Athanasius ausgehend als typisch geltende »arianische« Positionen formuliert: 79

Bekenntnis des Athanasius:

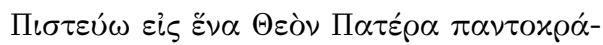

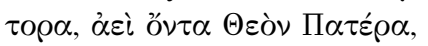

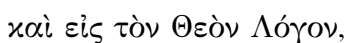

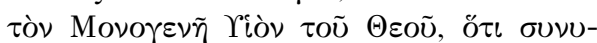

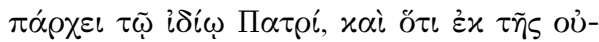

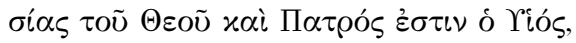

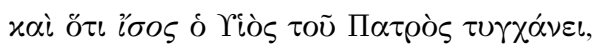

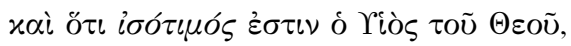

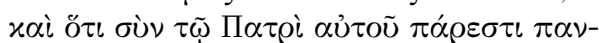

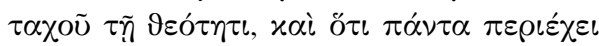

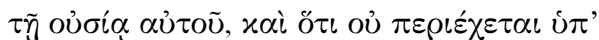

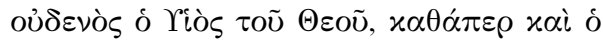

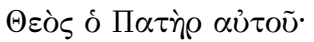

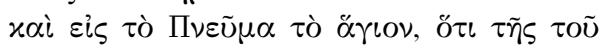

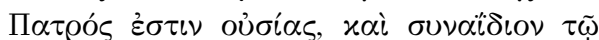

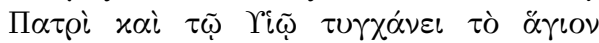
$\Pi \nu \varepsilon \tilde{u} \mu \alpha$.

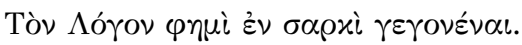

Bekenntnis des Arius:

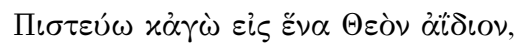

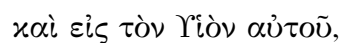

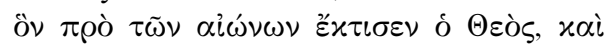

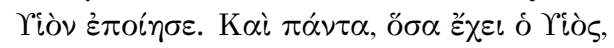

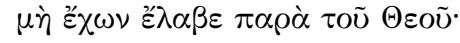

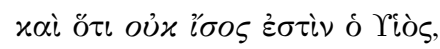

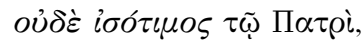

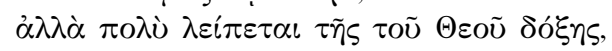

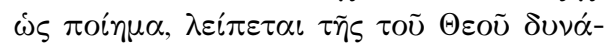
$\mu \varepsilon \omega \varsigma$

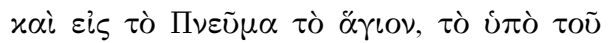
rioũ rełovós.

77 Dies ließe sich aus der Erzähllogik des Dialogs natürlich so erklären, daß das Gespräch zwischen Arius und Athanasius vor der Verabschiedung des Nizänums stattgefunden habe. Allerdings ist der Text auch insofern nicht realitätsnah, als sich in ihm Arius ja am Schluß bekehrt (Kap. 45). Die Disputatio contra Arium bietet insofern eben eine phantasievolle Darstellung eines virtuellen Verlaufs der Synode von Nizäa, so daß selbst solche Texte wie ein Bekenntnis den Bedürfnissen des Dialogs folgend abgewandelt werden können.

78 Disp. 6 (PG 28,444A); der griechische Text folgt in der tabellarischen Übersicht unten.

79 Sehr deutlich läßt sich das an den beiden durch Kursivierung gekennzeichneten Stellen zeigen. 
Auf der Basis dieser beiden Bekenntnisse (oder besser auf Basis des Bekenntnisses des Athanasius) setzt nun die eigentliche Diskussion ein. In ihr werden die Standardthemen des arianischen Streites in mehreren »Runden« abgehandelt:

Arius betont, daß Gott ewig ist, daß allein er Gott ist, daß der Sohn von Gott

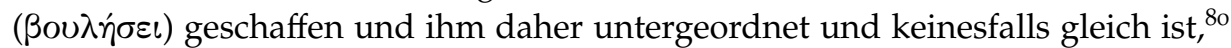

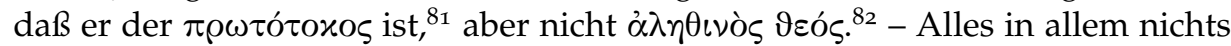
Überraschendes, sondern das, was von »nizänischer « Seite an theologischen Aussagen von einem Arianer erwartet werden würde.

Hier werden auf jeden Fall keine theologischen Positionen neu ent- oder alte weiterentwickelt. Wenn man den Dialog liest und sieht, wie in ihm die Diskussion geführt wird, so wird sehr deutlich, daß er keine theologische Programmschrift oder sonst ein Text ist, durch den die theologische Diskussion vorangetrieben werden wollte oder sollte. Hier positioniert sich keiner der beiden Disputanten in einer produktiven Diskussion.

Das zeigt sich in gleicher Weise an den von Athanasius vertretenen Positionen. Auch von ihm wird nur Altbekanntes wiederholt: Der Sohn existiert zusammen mit dem Vater, ${ }^{83}$ er ist zusammen mit ihm ewig, ${ }^{84}$ er ist aus dem Wesen des Vaters, er ist wahrhaftiger Gott. ${ }^{85}$

Charakteristisch für den gesamten Diskussionsverlauf ist aber, daß er ganz und gar von der Interpretation der loci classici des arianischen Streites seine Prägung erhält: Auf arianischer Seite sind das 1Kor 8,6, Joh 5,19, Joh 14,28, Prov 8,22, Apg 2,36, Joh 18,3, Joh 15,1 und Amos 4,13, auf athanasianischer Seite 1Kor 1,24, Joh 10,30, Joh 14,9, Röm 1,20, 1Joh 5,20, Joh 1,1, Hebr 2,10 und Mt 28,19. ${ }^{86}$

Beide Seiten berufen sich auf die Bibel, kommen aber zu einander entgegengesetzten Positionen. Daher ist im Prinzip fast die gesamte Diskussion ab Kap. 10 - in Kap. 7-9 findet ein erster Austausch statt, in dem die dort diskutierten Stellen einfach adversativ einander gegenüber gestellt werden - ein exegetischer Durchgang, durch den erklärt werden soll, wie sich die (scheinbar) bestehenden Widersprüche zwischen den Referenztexten auflösen lassen, d.h. wie die von den Arianern angeführten Bibelstellen in nizänischem Sinne verstanden werden können, nämlich so, daß die Niedrigkeitsaussagen durchgehend nicht auf den Logos, sondern auf den Inkarnierten bezogen werden. Dabei ist das Axiom, daß Gott sich nicht selbst widersprechen kann, ausschlaggebend und wird auch explizit thematisiert. ${ }^{87}$

80 Disp. 7 (PG 28,444BC).

81 Disp. 15 (PG 28,453B).

82 Disp. 22 (PG 28,464C)

83 Disp. 5 (PG 28,441D).

84 Disp. 13 (PG 28,452AB).

85 Disp. 30 (PG 28,477BC). An dieser Stelle fällt dann sogar doch noch das Stichwort ónooú

86 Vgl. die Anm. in PG 28,440-501.

87 Disp. 9 (PG 28,448A). 
Betrachtet man sich die angeführten Bibelverse und ihre Auslegung im Einzelnen sowie den Gang der Argumentation insgesamt, so fällt auf, daß sie weitestgehend dem Aufbau und der Argumentation der drei athanasianischen Arianerreden folgt. ${ }^{88}$ Aus dieser großen Nähe zu den Arianerreden wird dann eventuell auch verständlich, warum sich in der Disputatio contra Arium so gut wie keine Rezeptionsspuren des Nizänums finden lassen. Denn auch in den Arianerreden wird das Nizänum nicht angeführt, wie sich Athanasius ja überhaupt erst sehr spät, nämlich Ende der 5oer Jahre, direkt und explizit auf das Nizänum (bzw. seine beiden Kernbegriffe) bezieht, bzw. das Nizänum bei ihm erst sehr spät zur absoluten Norm für Orthodoxie wird. ${ }^{89}$ So verwundert es nicht, daß auch andere spätere Entwicklungen nicht rezipiert sind; denn von

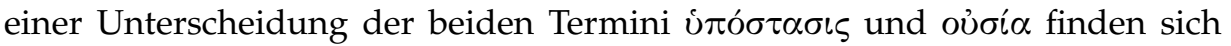
ebenfalls keine Anzeichen.

Im Gegensatz zu den Arianerreden wird jedoch die Diskussion in der Disputatio contra Arium insgesamt weniger aggressiv geführt. Zwar ist die Disputation passagenweise von durchaus aggressiven und fast stakkatohaft vorgetragenen Fragen »ja oder nein? « ( $\nu \alpha \iota \hat{\imath} \hat{\eta}$ oü;) geprägt, ${ }^{90}$ mit denen Arius von Athanasius regelrecht in die Enge getrieben wird, aber der Umgang der beiden Disputanten untereinander ist grundsätzlich ein sehr kultivierter: Arius ist in der Disputatio

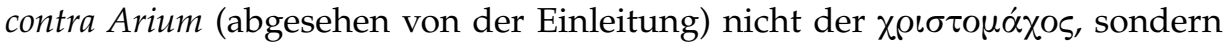
nur der theologisch etwas Minderbemittelte, der im Übrigen so agiert, wie man es sich vom »Junior «-Disputationspartner in einem sokratischen Dialog vorstellt, d.h. der erst eine andere Meinung vertritt, sich aber dann relativ schnell überzeugen lässt und zudem die Fragen des Athanasius meist nicht beantworten kann.

Auf dem Hintergrund dieses ziemlich offensichtlich zugrunde liegenden Modells eines sokratischen Dialogs verwundert es auch nicht, daß die Redeanteile des Athanasius umso größer werden, je weiter der Dialog fortschreitet..$^{91}$

Nachdem dann die trinitätstheologischen Bibelstellen allesamt abgehandelt und in nizänischer Weise interpretiert worden sind, wird in einem weiteren, abschließenden Teil, der die Kapitel 37-44 umfaßt, schließlich auch die Frage nach der Pneumatologie und damit verbunden die Frage nach der Trinität generell verhandelt.

88 Das zu zeigen ist einer weiteren Untersuchung vorbehalten, aber es ist ganz frappierend, wie sehr die Disputatio contra Arium gerade in den Einzelheiten von den Arianerreden abhängig ist.

89 Vgl. Stockhausen, Athanasius von Alexandrien. Epistula ad Afros.

90 Vgl. disp. 8 (PG 28,445B); 11 (PG 28,449A); 16 (PG 28,456B); 19 (PG 28,460D); 20 (PG 28,461B); 21 (PG 28,464A); 22 (PG 464D); 23 (PG 28,465D.468A); 24 (PG 28,468A); 25 (PG 28,469B); 30 (PG 28,477A.C); 31 (PG 28,480BC); 39 (PG 28,489C.492AB); 40 (PG 28,493A); oftmals findet sich im

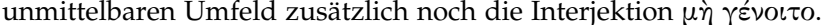

91 Arius ist insgesamt oft nur Stichwortgeber. Der Redeüberschuß des Athanasius wird aber besonders deutlich ab Kap. 25 generell und in besonderem Maße in Kap. 32-36. 
Wie bereits im vorangehenden Diskussionsgang über die Zuordnung des Logos im Rahmen der Gottheit, greift der Verfasser der Disputatio contra Arium auch jetzt in seiner Darlegung wieder ganz auf athanasianisches Gut zurück, nun, da es um die Pneumatologie geht, in erster Linie auf die dritte Arianerrede.

Zur Aufnahme des Vorbildes des sokratischen Dialogs und zu dem schon vermerkten eher konzilianten Gesprächsklima passt schließlich wiederum auch, daß sich Arius am Ende der Disputation (Kap. 45-46) von seiner Häresie abund dem nizänischen Glauben endgültig zuwendet, nachdem er im Laufe der Diskussion schon Schritt für Schritt von seinen häretischen Meinungen Abschied genommen hatte.

Der Aufbau des Dialogs ist zusammengefaßt also folgender:

$\begin{array}{ll}\text { 1-3 } & \text { Szenische Einleitung } \\ 4-6 & \text { Bekenntnisse von Athanasius und von Arius } \\ 7-9 & \begin{array}{l}\text { Biblische Texte über das Verhältnis von Vater } \\ \text { und Sohn }\end{array} \\ \text { 10-36 } & \begin{array}{l}\text { Wie die Widersprüche zwischen den bibli- } \\ \text { schen Texten aufgelöst werden können }\end{array} \\ 37-44 & \text { Über den Hl. Geist und die Trinität } \\ 45-46 & \text { Schluß }\end{array}$

Auffällige und unstimmige Elemente im Text

Nach diesem knappen Überblick über den Aufbau und die Inhalte der Disputatio contra Arium möchte ich nun noch einige auffällige und für die Interpretation des Textes interessante bzw. schwierige Passagen wenigstens kurz nennen:

Der Dialog hat - geht man nach dem handschriftlich überlieferten Titel auf der Synode von Nizäa stattgefunden. Ich habe bereits gesagt, daß sich im Text selbst dafür keinerlei Anhaltspunkt finden lassen. Nizäa wird im Text kein einziges Mal genannt, ebenso wenig wie überhaupt auf die Situation einer Synode angespielt wird. Die einleitend dargestellte Szenerie erinnert jedenfalls eher an einen Philosophenzirkel, der umherwandelnd verschiedene Fragen diskutiert.

Zudem sagt Athanasius in der szenischen Einleitung, daß die Gruppe langsam gegangen sei »wegen der Schwäche des Körpers.$^{92}$ Diese Aussage passt natürlich gar nicht zu dem doch noch jungen Mann Athanasius, der als Diakon seinen Bischof Alexander nach Nizäa begleitet hat.

Außerdem ist der Dialogpartner des Athanasius im Text immer als "Arei-

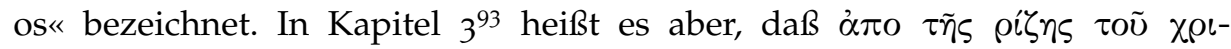

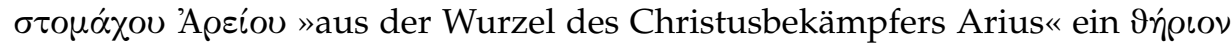

92 Disp. 1 (PG 28,440A).

93 Disp. 3 (PG 28,441B). 
$\dot{\alpha} \nu \theta \rho \omega \pi$ ó $\mu \rho \varphi \varphi \nu$ ein »menschengestaltiges Tier« Athanasius zur Disputation auffordert, also offenbar nicht Arius selbst.

Ein weiterer interessanter Text findet sich in Kap. $22 .{ }^{94} \mathrm{In}$ ihm bringt Athanasius zur Verdeutlichung seiner Argumentation, daß auch der Sohn »wahrer Gott« ist und daß Joh 17,3 in diesem Sinne zu verstehen ist, ein Beispiel.

Arius wollte den Vers nämlich so verstehen, daß nur Gott »wahrhaftiger

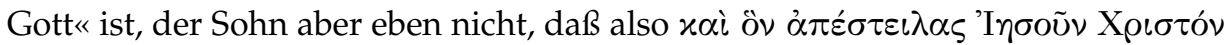

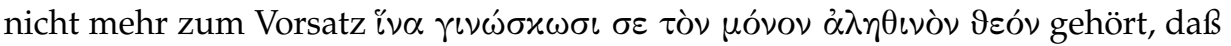
also das $x \alpha i$ kein additives $x \alpha i$ ist. Athanasius hält daraufhin Arius entgegen, daß es sich um einen $\sigma 0 \nu \delta \varepsilon \sigma \mu o ́ s ~ h a n d e l t$, die beiden Satzteile also zusammen zu betrachten und keinesfalls voneinander zu trennen sind. Und zur Verdeutlichung seines Argumentes führt er nun folgenden Vergleich an:

»Wenn einer seinem Nächsten über Konstantin erzählt und sagt: Erkenne sicher den einzigen Augustus und Selbstherrscher der Erde und des Meeres, den Kaiser Konstantin und seinen Sohn Konstantius! Bekennt er dann den Sohn zusammen mit dem Vater? Ja oder nein?« 95 Woraufhin Arius antwortet: "Darin liegt keine geringe Gefahr, wenn man sagt, daß Konstantius nicht mit seinem Vater Konstantin herrscht, indem man ihn unter ihn subsummiert.« ${ }^{96}$ Und daraufhin kontert Athanasius: »Bei Konstantin und Konstantius siehst du die größte Gefahr, bei Jesus Christus aber und seinem Vater fürchtest du dich nicht, wenn du solches äußerst. Du musst eine eben solche Verehrung dem Herrn gegenüber aufbringen, wie auch gegenüber dem genannten Konstantin. Du raubst nichts von seinem Sohn Konstantius, das heißt, daß er Augustus ist, und alles, was bezüglich seines Vaters vorher gesagt wurde, indem du nirgends anders die Einheit der Würde erkennst als durch ihn.«97

Bernhard Voss ${ }^{98}$ hat überlegt, ob sich hieraus nicht ablesen ließe, daß der Dialog doch sehr früh entstanden ist und daß hier somit echtes zeitgenössisches Wissen vorliegt. Dies scheint mir unwahrscheinlich, da hier ja im Prinzip eine Situation vor Augen gestellt wird, in der von den drei Konstantin-Söhnen nur noch Konstantius übrig geblieben ist, da nur und gerade er in dem Vater-Sohn-

94 Disp. 22 (PG 28,464CD).

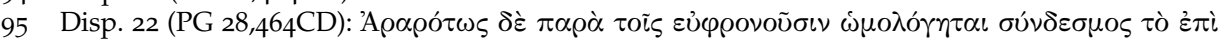

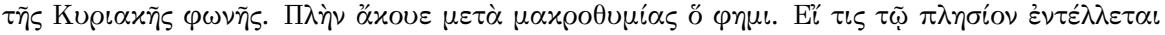

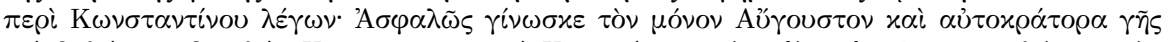

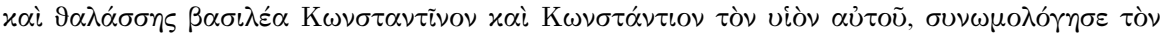

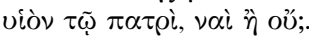

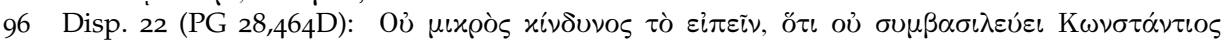

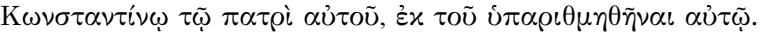

97 Disp. 22 (PG 28,464D-465A): 'E

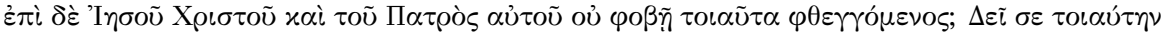

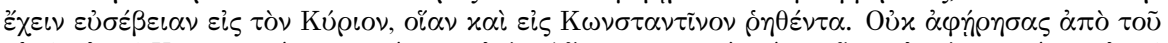

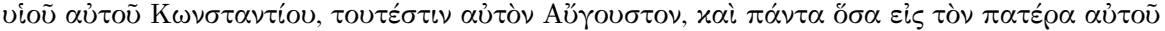

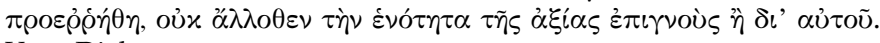


Vergleich angeführt wird, womit dieser Vergleich frühestens nach dem Jahr 350 formuliert sein kann. Mir scheint daher eher gelehrtes Wissen vorzuliegen, das hier vom Autor angebracht wird: Daß von den Konstantin-Söhnen letztlich nur der allein überlebende Konstantius eine wichtige Rolle in der Geschichte der Kirche gespielt hat. ${ }^{99}$ Solches gelehrtes Wissen des Verfassers läßt sich auch noch an einigen anderen Stellen beobachten: So wird in Kap. 20 ein weiterer Vergleich mit dem Kaiser angeführt, in Kap. 25 f. ein sehr detailliert ausgebauter Vergleich über den Hausbau und ebenfalls in Kap. 25 schließlich noch ein Vergleich über die Kalender-Berechnung anhand des Mondlaufes.

\section{Autorschaft und Datierung}

So bleibt zum Schluß noch die Frage, wie nun der Text eigentlich zeitlich anzusetzen ist und ob er sich dann gegebenenfalls sogar einem Autor zuschreiben läßt.

Geht man von seinem Titel aus, so ist der Dialog die Niederschrift einer Disputation zwischen Athanasius und Arius während der Synode von Nizäa. Wie wir bereits gesehen haben, spricht nichts dafür, daß diese Einordnung richtig ist. Die trinitätstheologische Diskussion des 4. Jahrhunderts ist sehr pauschal wiedergeben. Nizäa spielt keine Rolle, aber auch die weiteren großen Synoden des 4. Jahrhunderts, wie die Synode von Serdika oder die Synoden von Rimini, Seleukia und Konstantinopel, kommen ebenso wenig vor wie die durch das Aufkommen der neuarianischen oder homöischen Theologie neu zu bearbeitenden Probleme. Ein aktueller Anlaß für den Dialog lässt sich nicht einmal von Ferne erkennen. Eine zeitliche Einordnung auf diesem Wege ist daher sehr schwierig bzw. eigentlich unmöglich.

Ich möchte dennoch eine Datierung vorschlagen und es vor allem auch wagen, einen Verfasser zu vermuten:

Ein wichtiger, zwar selbstverständlicher, aber dennoch grundsätzlicher Hinweis ist natürlich der Umstand, daß die Schrift unter dem Namen des Athanasius überliefert ist. Sie gehört also auf jeden Fall, auch wenn sie sicher nicht von Athanasius selbst stammt, in den Umkreis der athanasianischen Tradition. Aus diesem Grund legen sich natürlich zuerst einmal die »üblichen Verdächtigen« nahe, d.h. Markell, Apolinaris oder die Alt-Nizäner in Antiochien, deren Schriften zum Teil unter dem Namen des Athanasius überliefert sind. Doch auch hier bliebe das Problem, daß ein aktueller Anlaß der Schrift nicht zu erkennen ist, der grundsätzlich einen der Autoren des trinitarischen Streites wahrscheinlich machen würde, und wie es auf die anderen Pseud-Athanasiana aus dieser Gruppe zutrifft.

99 Außerdem ist zu bedenken, daß die Arianerreden, die ja gewissermaßen dem Autor der Disputatio contra Arium als Vorlage dienten, zu Lebzeiten des Konstantius abgefaßt worden sind, vgl. Ar. I 10. 
Man kann aber aus der deutlichen Rezeption der Arianerreden in der Disputatio contra Arium durchaus feststellen, daß in ihr ein athanasianisches Coleur zu erkennen ist, d.h. annehmen, daß der Text in Alexandrien verfasst wurde, wo das Erbe des Athanasius zu allen Zeit besonders wertgeschätzt und bewahrt wurde.

Dazu kommt ein anderer Aspekt: Die Disputatio lässt sich, wie wir gesehen haben, als ein Text charakterisieren, der keine neuen Gedanken in die theologische Diskussion einbringt, sondern vielmehr alte, bereits ausgeführte Gedanken und Argumentationsmuster neu formuliert. Dabei ist der Autor aber nicht jemand, der mehr schlecht als recht von Athanasius schon einmal Geschriebenes nachschreibt, sondern er ist jemand, der die Gedanken des Athanasius in eine neue literarische Form, nämlich die des (sokratischen) Dialogs, bringt und der dies durchaus mit einigem Witz tut.

Dies kann man daran erkennen, daß er sein Opus versteckt als literarisches Übungsstück kennzeichnet, wenn er zu Beginn, in der oben zitierten Stelle aus Kap. $1^{100}$ den Vers 1 Tim 4,7 an einer sehr herausgehobenen Stelle zitiert: »Übe dich selbst hin zur Frömmigkeit! «101

Es gibt nun einen alexandrinischen Autor (und zugleich Nachfolger des Athanasius), mit dem sich diese Charakteristika in Zusammenhang bringen lassen, und das ist Kyrill von Alexandrien. Denn dieser hat - sehr wahrscheinlich bevor er Bischof wurde - zwei in ihrer Tendenz vergleichbare Werke verfasst, die unter seinem eigenen Namen überliefert sind: Den Thesaurus de trinitate und die De trinitate dialogi.

Auf die Disputatio contra Arium trifft genau zu, was Thomas Graumann in seiner Habilitationsschrift »Die Kirche der Väter« über Kyrill schreibt:

So spricht vieles dafür, daß sich Kyrill in Jugendjahren und wohl auch noch zu Anfang seines Episkopats auf den erreichten Stand der theologischen Diskussion und Lehrbildung in der Weise zu bringen versuchte, daß er wichtige Werke der Literatur des zurückliegenden Jahrhunderts studierte und gleichsam zur Übung in einer ähnlichen Weise literarisch um- und nacharbeitete, wie es auch im schulischen Unterricht in den Progymnasmata gängig war. ${ }^{102}$

Für Kyrill als Autor sprechen darüber hinaus auch einige sprachliche Anhalts-

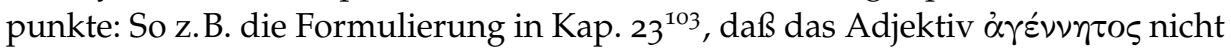

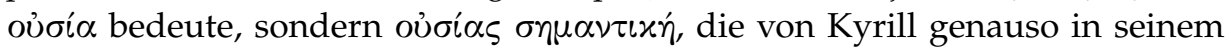

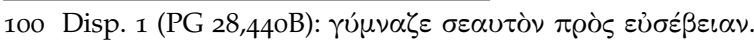

101 Zum Übungscharakter der späteren platonisch-sokratischen Dialoge vgl. Gundert, Dialog und Dialektik. Zur Struktur des platonischen Dialogs, $99 \mathrm{f}$.

102 Thomas Graumann, Die Kirche der Väter (Beiträge zur historischen Theologie 118), Tübingen 2002, 260 .

103 Disp. 23 (PG 28,465C). 
Thesaurus verwendet wird. ${ }^{104}$ Oder auf anderer, sprachlicher Ebene zeugt auch

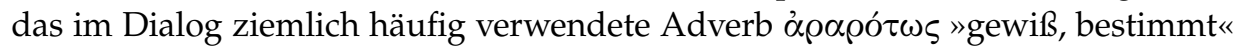
davon, da es gewissermaßen ein kyrillisches Lieblingsadverb ist.

Daß Werke Kyrills unter dem Namen des Athanasius überliefert wurden, zeigt außerdem die »Oratio in ascensionem domini $\ll^{105}$. Es ist also auch aus diesem Grund nicht völlig unwahrscheinlich, daß die pseud-athanasianische »Disputatio contra Arium « ein Werk des Kyrill von Alexandrien ist. ${ }^{106}$

Eine weitere Untersuchung des Textes kann hier wahrscheinlich noch weitere Belege beibringen, aber selbst wenn sich die kyrillische Verfasserschaft letztlich nicht wird erhärten lassen können, so muß es zumindest ein Autor sein, auf den die hier angeführten Charakteristika zutreffen.

Sollte aber Kyrill von Alexandrien wirklich der Autor der Disputatio contra Arium sein und sollte zugleich der Text einmal wirklich Teil der $x \alpha \tau \grave{\alpha}$ 'Apsíov

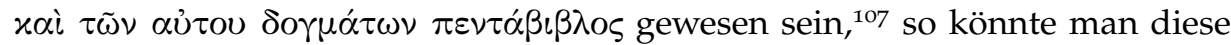
wahrscheinlich erste Sammlung der antiarianischen Schriften in Alexandrien verorten und eventuell sogar noch Kyrill selbst zuschreiben.

\section{Fazit}

Texte wie der hier vorgestellte und besprochene werden in der Forschung meist vernachlässigt. Hauptgrund dafür ist natürlich, daß sie als unecht gelten und daher dann auch als uninteressant, weil sie zu unserem Bild des Athanasius von Alexandrien und seiner Theologie nichts beitragen können. Dazu und infolgedessen kommt dann noch, daß sie in keiner Übersetzung in irgendeine modernen Sprache vorliegen, und aus diesem Grund dann natürlich erst recht nicht zur Kenntnis genommen werden - jedenfalls nicht in einem Umfang, der über eine Fußnote hinausginge.

Ich hoffe, daß ich mit den hier vorgeführten rudimentären Ausführungen zeigen konnte, daß Texte wie der hier besprochene dennoch einer näheren Untersuchung wert sind, auch wenn sie selbst keinen eigenen theologischen Beitrag leisten, sondern »nur « von der Rezeption und traditionsbildenden Aneignung wichtiger Theologen wie Athanasius zeugen.

Zugleich sollte dieser Beitrag ein erster Versuch im Rahmen eines noch auszuarbeitenden Projektes sein, das die Edition der pseud-athanasianischen Schriften und Untersuchungen über diese Werke zum Inhalt hat.

104 Cyr., thes. 3 .

105 CPG 2171=5281, vgl. C. Datema, Une homélie inédite sur l'ascension, Byzantion 44 (1974), 126-137.

106 Wenn auch im Falle der Disputatio contra Arium die Falschzuschreibung eher darauf zurückzuführen ist, daß der Name des Protagonisten des Dialoges auf den Autor überging, vgl. oben Anm. 28.

107 Vgl. oben S. $140 \mathrm{f}$. 


\section{Literatur}

Amidon, Philip R., The church history of Rufinus of Aquileia. Books 10 and 11, Oxford/New York 1997.

Batiffol, Pierre, Les sources de l'histoire du concile de Nicée, Échos d'Orient 24 (1925), 385-402; 30 (1927), 5-17.

Bekker, Immanuel, Nicephori Gregorae Byzantina historia III (CSHB), Bonnae 1855.

- Photii Bibliotheca I, Berlin 1824.

Bizer, Christoph, Studien zu pseudathanasianischen Dialogen der Orthodoxos und Aëtios, Diss., Bonn: theol., 1970.

Brennecke, Hanns Christof, Nicäa I. Ökumenische Synode von 325, TRE 24 (1994), 429-441.

Brennecke, Hanns Christof, Uta Heil und Annette von Stockhausen, Athanasius Werke II 8. Die »Apologien«, Berlin/New York 2006.

Brennecke, Hanns Christof, Uta Heil, Annette von Stockhausen und Angelika Wintjes, Athanasius Werke. Band III/Teil 1: Dokumente zur Geschichte des arianischen Streites. Lfg 3: Bis zur Ekthesis Makrostichos, Berlin/New York 2007.

Cañellas, Juan Nadal, Gregorii Acindyni refutationes duae operis Gregorii Palamae cui titulus Dialogus inter orthodoxum et Barlaamitam (CChr.SG 31), Turnhout 1995.

Casey, Robert Pierce, Armenian Manuscripts of St. Athanasius of Alexandria, HThR 25 (1932), 43-59.

Chadwick, Henry, The Origin of the Title »Oecumenical Council«, JThS 23 (1972), 132-135.

Chrysos, Evangelos, Konzilsakten und Konzilsprotokolle vom 4. bis 7. Jahrhundert, AHC 15 (1983), 30-40.

Datema, C., Une homélie inédite sur l'ascension, Byzantion 44 (1974), 126-137.

Declercq, Georges, Anno Domini. The origins of the Christian era, Turnhout 2000.

Ficker, Gerhard, Studien zu Vigilius von Thapsus, Leipzig 1897.

Geerard, Maurice, Clavis patrum Graecorum. II Ab Athanasio ad Chrysostomum (Corpus christianorum), Turnhout 1974.

Geerard, Maurice und Jacques Noret, Clavis patrum Graecorum. VI Supplementum (Corpus christianorum), Turnhout 1998.

Gelzer, Heinrich, Heinrich Hilgenfeld und Otto Cuntz (Hrsg.), Patrum Nicaenorum nomina Latine, Graece, Coptice, Syriace, Arabice, Armeniace (Bibliotheca scriptorum Graecorum et Romanorum Teubneriana), Stuttgart/Leipzig 1995. 
Gill, Joseph, Quae supersunt actorum Graecorum Concilii Florentini. Pars II Res Florentiae gestae (Concilium Florentinum. Documenta et scriptores B V 2), Roma 1953.

Girardet, K. M., Kaisergericht und Bischofsgericht. Studien zu den Anfängen des Donatistenstreites (313-315) und zum Prozeß des Athanasius von Alexandrien (328-346) (Antiquitas I 21), Bonn 1975.

Graumann, Thomas, Die Kirche der Väter (Beiträge zur historischen Theologie 118), Tübingen 2002.

Gundert, Hermann, Der platonische Dialog (Bibliothek der klassischen Altertumswissenschaften. Neue Folge 2,26), Heidelberg 1968.

- Dialog und Dialektik. Zur Struktur des platonischen Dialogs (Studien zur antiken Philosophie 1), Amsterdam 1971.

Hansen, Günther Christian, Anonyme Kirchengeschichte (Gelasius Cyzicenus, CPG 6034) (GCS.NF 9), Berlin 2002.

Jugie, M., La dispute des philosophes paiens avec les pères de Nicée, Échos d'Orient 24 (1925), 403-410.

Martin, Annick, Athanase d'Alexandrie et l'eglise d'Egypte au IV siècle (328373), Rom 1996.

Metzler, Karin, Kontamination in der Athanasius-Überlieferung, REByz 48 (1990), 213-232.

Metzler, Karin, Dirk Uwe Hansen und Kyriakos Savvidis, Athanasius Werke. Band I/Teil 1: Die Dogmatischen Schriften. Lfg. I Epistula ad episcopos Aegypti et Libyae, Berlin/New York 1996.

- Athanasius Werke. Band I/Teil 1: Die Dogmatischen Schriften. Lfg. 2 Orationes I et II contra Arianos, Berlin/New York 1998.

Metzler, Karin und Kyriakos Savvidis (Hrsg.), Athanasius Werke. Band I/Teil 1: Die Dogmatischen Schriften. Lfg. 3 Oratio III contra Arianos, Berlin/New York 2000.

Montfaucon, Bernard de, Sancti patris Nostri Athanasii Opuscula secundis curis reperta et antehac inedita (Collectio nova patrum et scriptorum graecorum, Eusebii Caesariensis, Athanasii, \& Cosmae Aegyptii), Paris 1706.

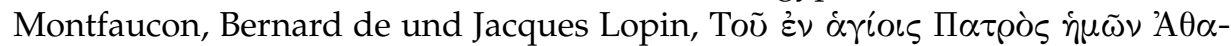

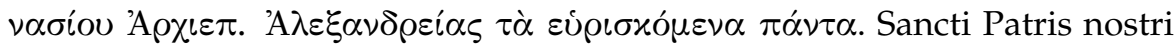
Athanasii Archiep. Alexandrini Opera omnia quae exstant vel quae ejus nomine circumferuntur, Ad mss. codices Gallicanos, Vaticanos, \&c. necnon ad Commelinianas lectiones castigata, multis aucta: nova Interpretatione, Praefationibus, Notis, variis lectionibus illustrata: novà Sancti Doctoris vitâ, Onomastico, \& copiosissimis Indicibus locupletata. Opera \& studio monachorum ordinis S. Benedicti è congretatione Sancti Mauri. Tribus Tomis in folio Graece et Latine, Paris 1698. 
Mosshammer, Alden A., The Easter computus and the origins of the Christian era (Oxford early Christian studies), Oxford 2008.

Müller, Dietram, Raum und Gespräch: Ortssymbolik in den Dialogen Platons, Hermes 116 (1988), 387-409.

Omont, Henri, Inventaire sommaire des manuscrits grecs de la Bibliothèque nationale I-IV, Paris $1886 / 1888 / 1898$.

Opitz, Hans-Georg, Athanasius Werke. Band II: Die Apologien. Lfg. 1-7, Berlin/Leipzig 1935-1941.

- Untersuchungen zur Überlieferung der Schriften des Athanasius (AKG 23), Berlin/Leipzig 1935.

Portmann, Werner, Athanasius, Zwei Schriften gegen die Arianer. Verteidigungsschrift gegen die Arianer / Geschichte der Arianer (BGL 65), Stuttgart 2006.

Renoux, Charles, Athanase d'Alexandrie dans le florilège arménien du Manuscrit Galata 54 (Deuxième Partie), Handes Amsorya 103 (1989), 7-27.

Schwartz, Eduard, Der s.g. Sermo maior de fide des Athanasius (SBAW.PPH 1924/6), München 1925.

- Drei dogmatische Schriften Iustinians (ABAW.PH 18), München 1939.

Stegmann, Anton, Die pseudoathanasianische »IVte Rede gegen die Arianer« als

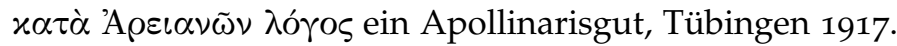

Stockhausen, Annette von, Athanasius von Alexandrien. Epistula ad Afros. Einleitung, Kommentar und Übersetzung (PTS 56), Berlin/New York 2002.

Thelamon, Françoise, Païens et chrétiens au IVe siècle. L'apport de l'« Histoire ecclésiastique » de Rufin d'Aquilée, Paris 1981.

Vinzent, Markus, Pseudo-Athanasius, contra Arianos IV. Eine Schrift gegen Asterius von Kappadokien, Eusebius von Cäsarea, Markell von Ankyra und Photin von Sirmium (SVigChr 36), Leiden [u.a.] 1996.

Voss, B. R., Der Dialog in der frühchristlichen Literatur, München 1970. 